\title{
IMPORTÂNCIA DO FONOAUDIÓLOGO NO ACOMPANHAMENTO DE INDIVÍDUOS COM HIPOTIREOIDISMO CONGÊNITO
}

\section{Speech and language pathologist importance in the attendance of individuals with congenital hypothyroidism}

Mariana Germano Gejão ${ }^{(1)}$, Amanda Tragueta Ferreira ${ }^{(2)}$, Dionísia Aparecida Cusin Lamônica (3)

\section{RESUMO}

Tema: o hipotireoidismo congênito é uma alteração metabólica que traz conseqüência graves para indivíduos não tratados e mesmo as crianças que realizam o tratamento podem apresentar distúrbios do desenvolvimento. O Programa Nacional de Triagem Neonatal, instituído pelo Ministério da Saúde, prevê o acompanhamento longitudinal de indivíduos com equipe multidisciplinar. Entretanto, a Fonoaudiologia não é incluída nesta equipe. Deste modo, considerando a ocorrência de distúrbios da comunicação nestes indivíduos, realizou-se levantamento bibliográfico nas bases de dados Lilacs, MedLine e PubMed, no período de 1987 a 2007, referente às alterações em habilidades do desenvolvimento decorrentes do hipotireoidismo congênito. Objetivo: verificar, na literatura científica, presença de alterações do desenvolvimento em indivíduos com hipotireoidismo congênito e refletir sobre a importância da atuação fonoaudiológica, em conjunto com equipe multidisciplinar especializada, no acompanhamento dos mesmos. Conclusão: a literatura relata alterações nas habilidades do desenvolvimento (motoras, cognitivas, lingüísticas e de autocuidados) e destaca que crianças com hipotireoidismo congênito são de risco para alterações no desenvolvimento lingüístico e, portanto, necessitam do acompanhamento longitudinal do desenvolvimento comunicativo. Torna-se evidente a importância da atuação do fonoaudiólogo nos Programas de Triagem Neonatal credenciados pelo Ministério da Saúde. Ressalta-se ainda a necessidade de investigações referentes às outras alterações metabólicas contempladas nestes programas, nas quais o fonoaudiólogo pode atuar de modo a prevenir, habilitar e reabilitar os distúrbios da comunicação, contribuindo para o trabalho em equipe, promovendo saúde nesta população.

DESCRITORES: Hipotireoidismo Congênito; Desenvolvimento Infantil; Desenvolvimento da Linguagem

(1) Fonoaudióloga; Laboratório de Screening Neonatal Teste do Pezinho da Associação de Pais e Amigos dos Excepcionais de Bauru; Mestre em Fonoaudiologia pela Faculdade de Odontologia de Bauru da Universidade de São Paulo. Fonoaudióloga; Clínica Spaço Vida.

(3) Fonoaudióloga; Professora Associada do Departamento de Fonoaudiologia da Faculdade de Odontologia de Bauru da Universidade de São Paulo; Livre-Docente em Distúrbios da Comunicação Humana pela Faculdade de Odontologia de Bauru da Universidade de São Paulo.

Trabalho premiado na XIV Jornada Fonoaudiológica de Bauru "Profa. Dra. Deborah Viviane Ferrari" em agosto de 2007. Faz parte da dissertação em Fonoaudiologia pela Faculdade de Odontologia de Bauru da Universidade de São Paulo, premiada no $5^{\circ}$ Congresso Brasileiro de Triagem Neonatal, $1^{\circ}$ Congresso de Erros Inatos do Metabolismo e $2^{\circ}$ Congresso Nacional de Triagem Neonatal em agosto de 2008.

\section{INTRODUÇÃO}

O hipotireoidismo congênito $(\mathrm{HC})$ é causado pela produção insuficiente dos hormônios tireoideanos devido à malformação da glândula tireóide ou alteração na biossíntese hormonal. É detectado em 1:4000 recém-nascidos e se tratado precocemente torna-se uma das poucas causas de retardo mental passível de prevenção ${ }^{1,2}$.

Durante a vida fetal e ao longo dos primeiros anos de vida, os hormônios tireoidianos exercem grande influência no desenvolvimento do sistema nervoso ${ }^{3,4}$. O desenvolvimento das habilidades cognitivas dos indivíduos diagnosticados com HC depende principalmente da precocidade do início 
do tratamento e adequação ao mesmo, além da gravidade da alteração metabólica ${ }^{3,5-7}$. Crianças tratadas tardiamente, sem controle hormonal e acompanhamento multidisciplinar adequados, tendem a apresentar alterações nas habilidades globais do desenvolvimento, ou seja, nas habilidades motoras, cognitivas, lingüísticas, sociais e de autocuidados além de retardo mental ${ }^{3,6,8-13}$.

A Triagem Neonatal (TN), também conhecida como Teste do Pezinho, detecta o HC e outras alterações congênitas do metabolismo. Deve ser realizada entre o terceiro e sétimo dia após o nascimento, permitindo o início do tratamento dentro do primeiro mês de vida e, conseqüentemente, a prevenção das seqüelas nas habilidades do desenvolvimento ${ }^{13}$.

Os centros credenciados do Ministério da Saúde por meio do Programas Nacional de Triagem Neonatal (PNTN), não inclui a atuação fonoaudiológica como parte da rotina de acompanhamento de crianças com HC. Estudos têm observado alterações do desenvolvimento da habilidade de linguagem em crianças com início do tratamento tardio e até precoce $^{3,6,14-19}$.

Diante do exposto, o objetivo do estudo foi verificar, na literatura científica, presença de alterações do desenvolvimento em indivíduos com $\mathrm{HC}$ e refletir sobre a importância da atuação fonoaudiológica, em conjunto com equipe multidisciplinar especializada, no acompanhamento desta população.

Para o desenvolvimento deste estudo, foram realizadas buscas da literatura pertinente nas seguin- tes bases de dados: Bireme (Medline e Lilacs) e Pubmed.

Os descritores de assunto e palavras chaves utilizadas nas bases de dados foram cruzados da seguinte forma: congenital hypothyroidism $(\mathrm{CH})$; $\mathrm{CH}$ AND child development; $\mathrm{CH}$ AND language development; $\mathrm{CH}$ AND cognition; $\mathrm{CH}$ AND language; $\mathrm{CH}$ AND motor development.

O critério de inclusão adotado para análise dos estudos científicos foi existência da correlação, no título ou resumo, de habilidades do desenvolvimento (motoras, cognitivas, lingüísticas e de autocuidados) e HC.

Os resultados do levantamento bibliográfico serão apresentados de maneira descritiva em forma de tabela.

\section{REVISÃO DA LITERATURA}

Enquadraram-se no critério de inclusão 38 estudos científicos. Destes, 31 avaliavam habilidades cognitivas, 18 motoras, 14 lingüísticas e quatro de autocuidados. Foi relatada ocorrência de alterações motoras em 15 estudos, lingüísticas e cognitivas em 11 e de autocuidados em quatro. Alguns estudos avaliaram mais de uma habilidade (Tabela 1).

Dos 38 estudos científicos analisados, a grande maioria (31) avaliou habilidades cognitivas ${ }^{3,7,14-18,20-32,35-44}$. A literatura refere grande preocupação no acompanhamento do desenvolvimento desta habilidade, uma vez que a principal seqüela do HC é o retardo mental, trazendo seqüelas

Tabela 1 - Estudos científicos analisados descritos de acordo com o ano de publicação, autores, habilidades do desenvolvimento avaliadas com presença ou ausência de alteração

\begin{tabular}{|c|c|c|c|c|c|c|c|}
\hline Ano & Autores & Habilidades & Conclusão & Ano & Autores & Habilidades & Conclusão \\
\hline 1987 & Rovet et al ${ }^{(20)}$ & $\mathrm{M} ; \mathrm{L} ; \mathrm{AC} ; \mathrm{C}$ & $\mathrm{M}+; \mathrm{L}+; \mathrm{AC}+; \mathrm{C}-$ & 2000 & Weber et al ${ }^{(29)}$ & $\mathrm{C}$ & $\mathrm{C}+$ \\
\hline 1990 & Bazán et al ${ }^{(8)}$ & M & $M_{+}$ & 2000 & Bongers-Schokking et al ${ }^{(30)}$ & C & $\mathrm{C}-$ \\
\hline 1990 & Rovet ${ }^{(21)}$ & C & C- & 2000 & Salerno et al ${ }^{(31)}$ & C & C- \\
\hline 1991 & Fuggle et al ${ }^{(22)}$ & $\mathrm{M} ; \mathrm{C}$ & $\mathrm{M}_{+}, \mathrm{C}_{+}$ & 2003 & Oerbeck et al ${ }^{(6)}$ & M; L; C & $\mathrm{M}+; \mathrm{L}+; \mathrm{C}+$ \\
\hline 1991 & Heyerdahl et al ${ }^{(23)}$ & C & C- & 2004 & Androvandi; Nunes ${ }^{(32)}$ & C & C- \\
\hline 1992 & Glorieux et al ${ }^{(24)}$ & C & $\mathrm{C}+$ & 2004 & Simons et al ${ }^{(33)}$ & $M ; L$ & M+; L- \\
\hline 1994 & Bragatti-Winckler; Rotta ${ }^{(9)}$ & $M ; L ; A C$ & $\mathrm{M}-; \mathrm{L}-; \mathrm{AC}+$ & 2004 & Alvarez et al ${ }^{(34)}$ & $M ; L$ & $M+; L+$ \\
\hline 1994 & Corrêa et al ${ }^{(10)}$ & $\mathrm{M} ; \mathrm{AC}$ & $\mathrm{M}+; \mathrm{AC}+$ & 2004 & Alvarez Gonzáles et al ${ }^{(35)}$ & $\mathrm{M} ; \mathrm{L} ; \mathrm{C}$ & $\mathrm{M}+; \mathrm{L}+; \mathrm{C}-$ \\
\hline 1994 & Gottschalk et al ${ }^{(25)}$ & $\mathrm{M} ; \mathrm{L} ; \mathrm{C}$ & $\mathrm{M}+; \mathrm{L}+; \mathrm{C}-$ & 2004 & Simoneal-Roy et al ${ }^{(36)}$ & C & C- \\
\hline 1994 & Kooistra et al ${ }^{(14)}$ & $\mathrm{M} ; \mathrm{L} ; \mathrm{C}$ & $\mathrm{M}+; \mathrm{L}+; \mathrm{C}+$ & 2005 & Bongers-Schokking et al ${ }^{(37)}$ & $\mathrm{C}$ & C- \\
\hline 1994 & Pizarro et al ${ }^{(11)}$ & $\mathrm{M}$ & M- & 2005 & Oerbeck et al ${ }^{(38)}$ & $\mathrm{C}$ & $\mathrm{C}+$ \\
\hline 1995 & Rovet; Ehrlich ${ }^{(15)}$ & $\mathrm{L} ; \mathrm{AC} ; \mathrm{C}$ & $\mathrm{L}+; \mathrm{AC}+; \mathrm{C}-$ & 2005 & Rovet $^{(7)}$ & C & C+ \\
\hline 1995 & Salerno et al ${ }^{(26)}$ & C & C- & 2006 & Bargagna et al ${ }^{(18)}$ & M; L; C & $\mathrm{M}+; \mathrm{L}+; \mathrm{C}-$ \\
\hline 1996 & Rovet; Alvarez ${ }^{(16)}$ & $\mathrm{L} ; \mathrm{C}$ & $\mathrm{L}+; \mathrm{C}-$ & 2006 & Dluholuck et al ${ }^{(39)}$ & C & C- \\
\hline 1998 & Manríquez et al ${ }^{(1)}$ & M & M- & 2006 & Pniewska-Siark et al ${ }^{(41)}$ & C & C- \\
\hline 1999 & Rovet $^{(3)}$ & $\mathrm{L} ; \mathrm{C}$ & $\mathrm{L}+; \mathrm{C}-$ & 2007 & Oerbeck et al ${ }^{(42)}$ & $\mathrm{C}$ & $\mathrm{C}+$ \\
\hline 1999 & Salerno et al ${ }^{(28)}$ & C & C- & 2007 & Nakamizo et al ${ }^{(43)}$ & $\mathrm{C}$ & C- \\
\hline 2000 & Bargagna et al ${ }^{(17)}$ & M; L; C & $\mathrm{M}+; \mathrm{L}+; \mathrm{C}-$ & 2007 & Kempers et al ${ }^{(44)}$ & $\mathrm{M} ; \mathrm{C}$ & $\mathrm{M}+; \mathrm{C}_{+}$ \\
\hline
\end{tabular}

Legenda: M: habilidades motoras; L: habilidades lingüísticas; AC: habilidades de autocuidados; C: habilidades cognitivas; +: alterações presentes; -: alterações ausentes. 
globais para o desenvolvimento, inclusive o desenvolvimento da linguagem ${ }^{12}$.

A habilidade motora foi a segunda habilidade do desenvolvimento estudada com mais freqüência. Dos 18 estudos científicos, 15 observaram alterações referentes a atraso na inibição dos reflexos primitivos, alterações do equilíbrio e da coordenação motora grossa e fina; atraso para o início da marcha, movimentos associados e alterações do tônus muscular 6,8-10,14,17-18,20,22,25,27,33-35,40,44.

Os hormônios tireoidianos estão envolvidos na maturação neuronal ${ }^{3,4}$ e na regulação das habilidades motoras ${ }^{17}$, o que pode justificar a possibilidade de alterações no desenvolvimento motor em indivíduos com HC. A criança adquire o conhecimento por meio da exploração do meio ambiente, manipulação dos objetos, repetição de ações, domínio próprio do controle do esquema corporal e relações estabelecidas nas situações por ela vivenciadas. Nestas circunstâncias, a criança com alteração motora pode ir perdendo as oportunidades concretas de viabilizar ampliações de seu repertório, podendo ocasionar lacunas nas áreas perceptivas, cognitiva, lingüística e social, com reflexos importantes para o desenvolvimento das habilidades de comunicação ${ }^{45}$.

Todos os 4 trabalhos que avaliaram o desenvolvimento de habilidades de autocuidados, observaram em crianças com diagnóstico de $\mathrm{HC}$ tardio, alterações nas funções sociais, atraso no controle de esfíncteres e dificuldades de sucção 9,10,15,20. Essas alterações podem ser decorrentes do atraso no desenvolvimento motor, cognitivo e lingüístico também presentes na maioria das crianças avaliadas pelos estudos.

Dos 14 trabalhos científicos que avaliaram habilidades lingüísticas, 11 observaram alterações. A literatura refere que indivíduos com $\mathrm{HC}$ apresentam com mais freqüência atraso para o início da linguagem oral, dificuldades de compreensão da linguagem, alterações articulatórias, fonológicas e morfossintáticas, vocabulário reduzido e dificuldade para nomeação 3,6,14-19,20,25,27,34,35.

Os achados deste estudo reforçam a necessidade da inserção do fonoaudiólogo na equipe de pro- fissionais dos Programas de Triagem Neonatal que acompanham longitudinalmente o desenvolvimento de indivíduos com $\mathrm{HC}$, uma vez estas crianças são de risco para alterações no desenvolvimento lingüístico e, portanto, necessitam do acompanhamento longitudinal do desenvolvimento comunicativo ${ }^{17,19}$. A importância desse acompanhamento tem sido ressaltada ${ }^{5,30,37}$ e, embora observado alterações de linguagem com o início do tratamento tardio e precoce, a atuação fonoaudiológica não é prevista na rotina de acompanhamento, segundo o Programa Nacional de Triagem Neonatal ${ }^{13}$.

Ressalta-se ainda a necessidade de investigações referentes às outras alterações metabólicas contempladas nestes programas, nas quais o fonoaudiólogo pode atuar de modo a prevenir, habilitar e reabilitar os distúrbios da comunicação, contribuindo para o trabalho em equipe, promovendo saúde nesta população.

\section{COMENTÁRIOS FINAIS}

A literatura relata alterações nas habilidades do desenvolvimento (motoras, cognitivas, lingüísticas e de autocuidados) e destaca que crianças com hipotireoidismo congênito são de risco para alterações no desenvolvimento lingüístico e, portanto, necessitam do acompanhamento longitudinal do desenvolvimento comunicativo. Torna-se evidente a importância da atuação do fonoaudiólogo nos Programas de Triagem Neonatal.

\section{AGRADECIMENTOS}

Agradecemos ao apoio financeiro da Fundação de Amparo a Pesquisa do Estado de São Paulo (FAPESP) e Conselho Nacional de Pesquisa (CNPq) e a colaboração da equipe de profissionais do Laboratório de Screening Neonatal Teste do Pezinho da Associação de Pais e Amigos dos Excepcionais de Bauru. 


\section{ABSTRACT}

Background: congenital hypothyroidism is a metabolic disturbance that causes severe consequence for non-treated individuals and even the children that accomplish the treatment can present development disturbances. The National Neonatal Screening Program, instituted by Health Ministry, foresees the individuals' longitudinal attendance with multidisciplinary team. However, the Speech and Language Pathologist is not included in this team. This way, considering the occurrence of communication disturbances in these individuals, a bibliographical assessment was carried out in Lilacs, MedLine and PubMed databases, in the period from 1987 to 2007, regarding the disturbances in development abilities arising from congenital hypothyroidism. Purpose: to check, in the scientific literature, for the presence of development alterations in individuals with congenital hypothyroidism and contemplate the importance of the speech and language pathologist performance, together with a multidisciplinary team specialized in their attendance. Conclusion: the literature reports disturbances in development abilities (motor, cognitive, linguistics and self-care) and stresses out that children with congenital hypothyroidism are under risk for disturbances in the linguistic development and, therefore, they need the longitudinal attendance of the communicative development. The importance of the speech and language pathologist performance in credential Neonatal Screening Programs by the Health Ministry becomes evident. It still fits to emphasize the need for investigations regarding the other metabolic alterations meditated in these programs, in which the speech and language pathologist can act in such a way to prevent, enable and rehabilitate the communication disturbances, contributing to the work in team, promoting health in this population.

KEYWORDS: Congenital Hypothyroidism; Child Development; Language Development

\section{REFERÊNCIAS}

1. Manríquez MO, Nagel LB, VivancoXW. Evaluación neurológica en pacientes con hipotiroidismo congénito diagnosticado por rastreo neonatal. Rev Chil Pediatr. 1998; 69(2):56-9.

2. Manglik AK, Chatterjee N, Ghosh G. Umbilical cord blood TSH levels in term neonates: a screening tool for congenital hypothyroidism. Indian Pediatr. 2005; 42(10):1029-32.

3. Rovet JF. Congenital hypothyroidism: long-term outcome. Thyroid. 1999; 9(7):741-8.

4. Nunes MT. Hormônios tireoidianos: mecanismo de ação e importância biológica. Arq Bras Encodrinol Metab. 2003; 47(6):639-43.

5. Almache ON. Hipotiroidismo congénito. Paediatr. 2003; 5(2):93-9.

6. Oerbeck B, Sunder K, Kase BF, Heyerdahl S. Congenital hypothyroism: influence of disease severity and I-thyroxine treatment on intellectual, motor, and school-associated outcomes in young adults. Pediatr. 2003; 112(4):923-30.

7. Rovet JF. Children with congenital hypothyroidism and their siblings: do they really differ? Pediatr. 2005; 115(1):52-7.

8. Bazan MC, Pasteris L, Chaila Z. Hipotiroidismo congenito y desarrollo mental. Cienc Méd (San Miguel de Tucuman). 1990; 5(6):189-96.
9. Bragatti-Winckler MI, Rotta NT. Hormônios tireóideos na sintomatologia de doenças neuropsiquiátricas. Arq Neuropsiquiatr. 1994; 52(3):427-30.

10. Corrêa AL, Breda DJ, Pereira GM, Schotkis MV, Feijó RB. Hipotireoidismo congênito: relato de caso. Pesq Med. 1994; 28(1):33-7.

11. Pizarro CB, Paskulin GA, Oliveira MC. Hipotireoidismo primário associado à puberdade precoce: um caso com apresentação incomum. Rev AMRIGS. 1994; 38(4):312-4.

12. Ludueña MP, Prada EMG, Sândi KP. Hipotiroidismo congênito: a propósito de um caso. Rev Soc Bol Ped. 2002; 41(1):11-4.

13. Brasil. Ministério da Saúde. Manual de normas técnicas e rotinas operacionais do programa nacional de triagem neonatal. Brasília: Ministério da Saúde; 2002. Disponível em URL: http:/www.saude. gov.br/sãs. Acesso em 25 de jul 2006.

14. Kooistra L, Laane C, Vulsma T, Schellekens $\mathrm{JMH}$, Van Der Meere JJ, Kalverboer AF. Motor and cognitive development in children with congenital hypothyroidism: a long-term evaluation of the effects of neonatal treatment. J Pediatr. 1994; 124(6):903-9.

15. Rovet JF, Ehrlich RM. Long-term effects of Ithyroxine therapy for congenital hypothyroidism. J Pediatr. 1995; 126(3):380-6. 
16. Rovet J, Alvarez M. Thyroid hormones and attention in children with congenital hypothyroidism. J Pediatric Endocrinol Metab. 1996; 9(1):63-6.

17. Bargagna S, Canepa G, Costagli C, Dinetti D, Marcheschi M, Millepiedi S, etal. Neuropsychological follow-up in early-treated congenital hypothyroism: a problem-oriented approach. Thyroid. 2000; 10(3):243-9.

18. Bargagna S, Astrea G, Perelli V, Rafanelii V. Neurophychiatric outcome in patients with congenital hypothyroidism precautiously treated: risk factors analysis in a group of patients from Tuscany. Minerva Pediatr. 2006; 58(3):279-87.

19. Gejão MG, Lamônica DAC. Habilidades do desenvolvimento em crianças com hipotireoidismo congênito: enfoque na comunicação. Pró-Fono. 2008; 20(1):25-30.

20. Rovet J, Ehrlich R, Sorbara D. Intellectual outcome in children with fetal hypothyroidism: implications for neonatal diagnosis. J Pediatr. 1987; 110(5):700-4.

21. Rovet JF. Does Breast-feeding protect the hypothyroid infant whose condition is diagnosed by newborn screening? Am J Dis Child. 1990; 144(3):319-23.

22. Fuggle PW, Grant DB, Smith I, Murphy G. Intelligence, motor skills and behaviour at 5 years in early-treated congenital hypothyroidism. Eur $\mathrm{J}$ Pediatr. 1991; 150(8):570-4.

23. Heyerdahl S, Kase BF, Lie SO. Intellectual development in children with congenital hypothyroisidm in relation to recommended thyroxine treatment. J Pediatr. 1991; 118(6):850-7.

24. Glorieux J, Dussault J, Van Vliet G. Intellectual development at age 12 years of children with congenital hypothyroidism diagnosed by neonatal screening. J Pediatr. 1992; 121(4):581-4.

25. Gottschalk B, Richman RA, Lewandowski L. Subtle speech and motor deficits of children with cogenital hypothyroidism treated early. Dev Med Child Neurol. 1994; 36(3):216-20.

26. Salerno M, Di-Maio S, Militerni R, Argenziano A, Valerio G, Tenore A. Prognostic factors in the intellectual development at 7 years of age in children with congenital hypothyroidism. J Endocrinol Invest. 1995; 18(10):774-9.

27. Bargagna S, Chiovato L, Dinetti D, Montanelli L, Giachetti C, Romolini E, et al. Neuropsychological development in a child with early-treated congenital hypothyroidism as compared with her unaffected identical twin. Eur J Endocrinol. 1997; 136(1):100-4.

28. Salerno M, Militerni R, Di Maio S, Bravaccio C, Gasparini N, Tenore A. Intellectual outcome at 12 years of age in congenital hypothyroidsm. Eur $\mathrm{J}$ Endocrinol. 1999; 141:105-10.
29. Weber G, Mora S, Prina Cerai LM, Siragusa V, Colombini J, Medaglini S, et al. Cognitive function and neurophysiological evaluation in earlytreated hypothyroid children. Neurol Sci. 2000; 21(5):307-14.

30. Bongers-Schokking JJ, Koot HM, Wiersma $\mathrm{D}$, Vernek $\mathrm{PH}$, de Muinck Keizer-Schrama SMPF. Influence of timing and dose of thyroid hormone replacement on development in infants with congenital hypothyroidism. J Pediatr. 2000; 136(3):292-7.

31. Salerno M, Di-Maio S, Micillo M, Officioso A, Ferri $P$, Lettiero $T$, et al. Effect of different starting doses of I-thyroxine on intellectual outcome in congenital hypothyroidism. Horm Res. 2000; 53(2 suppl.):16.

32. Androvandi C, Nunes MLT. Avaliação intelectual de escolares com hipotireoidismo congênito. Aletheia. 2004; (20):55-64.

33. Simons WF, Fuggle PW, Grant DB, Smith I. Educational progress, behavior, and motor skills at 10 years in early treated congenital hypothyroidism. Arch Dis Child. 2004; 77(3):219-22.

34. Alvarez M, Carvajal F, Renón A, Pérez C, Olivares A, Rodríguez G, Alvarez V. Differential effect of fetal, neonatal and treatment variables on neurodevelopment in infants with congenital hypothyroidism. Horm Res. 2004; 61(1):17-20.

35. Alvarez González MA, Carvajal Martínez F, Pérez Gesén C, Olivares Torres A, Fernández Yero $\mathrm{JL}$, Robaina Alvarez R, et al. [Prognosis of cognition in congenital hypothyroidism following early treatment. Double effect hypothesis]. Rev Neurol. 2004; 38(6):513-7.

36. Simoneau-Roy J, Marti S, Deal C, Huot C, Robaey P, Van Vliet G. Cognition and behavior at school entry in children with congenital hypothyroidism treated early with high-dose levothyroxine. J Pediatr. 2004; 144(6):747-52.

37. Bongers-Schokking JJ, de Muinck KeizerSchrama SM. Influence of timing and dose of thyroid hormone replacement on mental, psychomotor, and behavioral development in children with congenital hypothyroidism. J Pediatr. 2005; 147(6):768-74.

38. Oerbeck B, Sunder K, Kase BF, Heyerdahl S. Congenital hypothyroism: no adverse effects of high dose throxine treatment on adult memory, attention, and behaviour. Arch Dis Child. 2005; 90(2):132-7.

39. Dluholucky S, Hornová V, Lukác P. Congenital hypothyroidism in one of monozygotic twins: comparison of their long-term psychosomatic development. Neuro Endocrinol Lett. 2006; 27(1-2):203-8.

40. Kempers MJ, Van Der Sluijs Veer L, Nijhuis-Van Der Sander MW, Kooistra L, Wiedijk BM, Faber I, et al. Intellectual and motor development of young adults with congenital hypothyroidism diagnosed by 
neonatal screening. J Clin Endocrinol Metab. 2006; 91(2):418-24.

41. Pniewska-Siark B, Jeziorowska A, Bobeff I, Lewinski A. Analysis of physical and mental development of children with aplasia, hypoplasia and ectopy of the thyroid gland. Endocr Regul. 2006; 40(1):7-14.

42. Oerbeck B, Reinvang I, Sudent K, Heyerdalh S. Young adults with severe congenital hypothyroidism: cognitive event related potentials (ERPs) and the significance of an early start of thyroxine treatment. Scand J Psychol. 2007; 48(1):61-7.

43. Nakamizo M, Toyabe S, Asami T, Akazawa K. Mental development of infants with congenital hypothyroidism: a longitudinal study. Clin Pediatr. 2007; 46(1):53-8.
44. Kempers MJ, van der Sluijs Veer L, Nijhuis-van der Sanden RW, Lanting Cl, Kooistra L, Wiedijk $\mathrm{BM}$, et al. Neonatal screening for congenital hypothyroidism in the Netherlands: cognitive and motor outcome at 10 years of age. J Clin Endocrinol Metab. 2007; 92(3):919-24.

45. Lamônica DAC, De-Vitto LPM, Gejão MG. Introdução ao estudo do sistema nervoso e alterções do desenvolvimento que cursam com deficiência mental, deficiência física e transtornos invasivos do desenvolvimento. In: Genaro KF, Lamônica DAC, Bevilacqua MC. O processo de comunicação: contribuição para a formação de professores na inclusão de indivíduos com necessidades educacionais especiais. Campos do Jordão: Pulso; 2006. p. 23-42. 